\title{
Paravertebral Arteriovenous Fistula Treated by Endovascular Coil Embolization -Case Report-
}

\author{
Shinichiro TERAMOTO, ${ }^{1}$ Hidenori OISHI, ${ }^{1}$ Kensaku YOSHIDA, ${ }^{1}$ \\ Munetaka YAMAMOTO, ${ }^{1}$ Yukoh OHARA, ${ }^{1}$ and Hajime ARAI ${ }^{1}$ \\ ${ }^{1}$ Department of Neurosurgery, Juntendo University School of Medicine, Tokyo
}

\begin{abstract}
A 41-year-old man, without underlying health problems or traumatic episodes, presented with a rare paravertebral arteriovenous fistula (AVF) causing radiculopathy manifesting as gradually progressive right grip weakness, and right thumb, index, and middle finger numbness. Digital subtraction angiography revealed a high flow, single hole paravertebral AVF fed by the right thyrocervical trunk that drained into the epidural venous plexus. The patient underwent endovascular embolization of the AVF via the transarterial approach. The fistula was completely occluded by coil embolization. Postembolization, clinical symptoms improved and 6-month follow-up angiography showed no recurrence of the fistula. Paravertebral AVF is a rare vascular malformation occurring outside the dura mater, fed by dural or epidural branches, and draining primarily into the epidural venous plexus. Paravertebral AVF is usually asymptomatic because of a "reflux-impending mechanism" within the dural sleeves that prevents retrograde drainage into the perimedullary veins. However, in the present case, mechanical compression of the radicular nerve due to a dilated epidural venous plexus resulted in neurological symptoms. We conclude that endovascular surgery is an effective treatment strategy for paravertebral AVF.
\end{abstract}

Key words: paravertebral arteriovenous fistula, endovascular surgery, direct surgery, radiculopathy, thyrocervical trunk

\section{Introduction}

Spinal arteriovenous malformations (AVMs) are classified into 4 types, intramedullary AVM, perimedullary arteriovenous fistula (AVF), dural AVF, and paravertebral AVF occurring outside the dura. Spinal dural AVF is the most common type of spinal AVM, accounting for approximately $70 \%$ of cases. ${ }^{9)}$ Spinal dural AVF is a direct shunt located inside the dura mater between a radiculomeningeal artery and a radicular vein. Drainage is retrograde into the perimedullary veins through the radicular vein, and causes increasing venous pressure in the spinal cord leading to progressive myelopathy. ${ }^{9}{ }^{9}$ Direct or endovascular surgery, or a combination of both, are used for the treatment of spinal dural AVF. On the other hand, paravertebral AVF with epidural drainage rarely occurs and accounts for approximately $2.5-4 \%$ of all spinal AVMs. ${ }^{1,7)}$ Therefore, the therapeutic options of paravertebral AVF still remain controversial.

We present a case of paravertebral AVF manifesting as radiculopathy that was successfully treated with endovascular coil embolization.

\footnotetext{
Received March 10, 2011; Accepted August 31, 2011
}

\section{Case Report}

A 41-year-old man, without underlying health problems or traumatic episodes, presented with a history of gradually progressive right grip weakness and right thumb, index, and middle finger numbness. On admission, dilation of the superficial veins and pulsatile bruit were detected in the right side of his neck, and neurological examination revealed manual muscle testing grade IV weakness of the right upper limb and hypoesthesia in the C6, C7, and C8 areas on the right side. Radiography showed widening of the right C4-5 intervertebral foramen (Fig. 1A). Magnetic resonance imaging showed a large flow void in the right epidural space at the C4, C5, and C6 levels, which compressed the spinal cord without causing signal intensity change (Fig. 1B, C). Computed tomography (CT) with contrast medium also demonstrated abnormal enhancement indicating an enlarged vessel in the spinal canal at the $\mathrm{C} 4$, C5, and C6 levels (Fig. 1D, E). Three-dimensional CT angiography showed the right enlarged thyrocervical trunk entered the right C4-5 intervertebral foramen (Fig. 1F). Digital subtraction angiography also disclosed a paravertebral AVF fed by the right thyrocervical trunk in the right C4-5 intervertebral foramen, with a high flow shunt (Fig. 2A). The arterial feeder was enlarged, and no recruitment of the adjacent arteries was observed. Venous drainage 

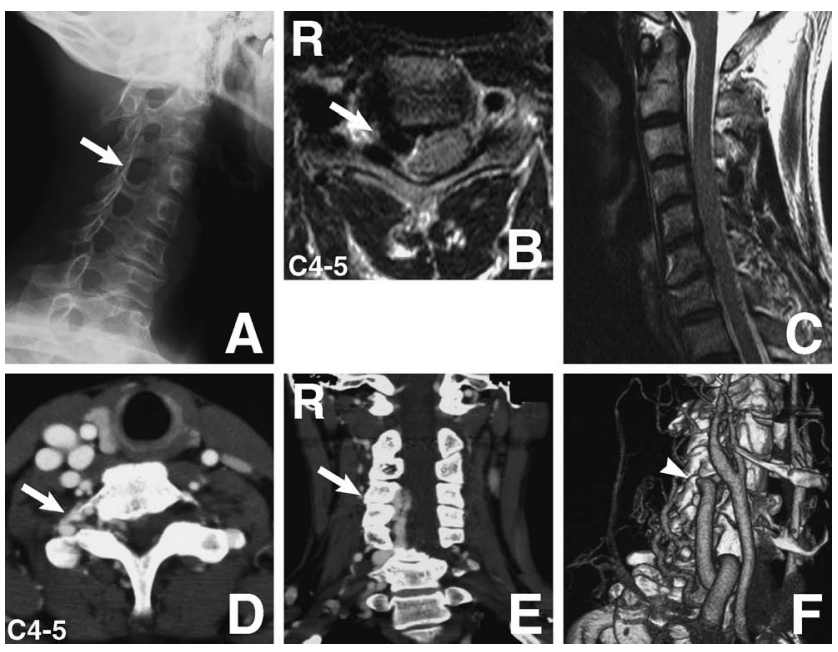

Fig. 1 A: Right anterior oblique radiograph showing widening of the right C4-5 intervertebral foramen (arrow). B: Axial $\mathrm{T}_{2^{-}}$ weighted magnetic resonance (MR) image showing a large flow void in the right epidural space at the $\mathrm{C4}, \mathrm{C5}$, and $\mathrm{C6}$ levels, compressing the spinal cord (arrow). C: Sagittal $\mathrm{T}_{2}$-weighted MR image demonstrating no signal intensity change in the spinal cord. D, E: Axial (D) and coronal (E) computed tomography (CT) scans showing an abnormal enhancement in the spinal canal at the $\mathrm{C} 4, \mathrm{C} 5$, and $\mathrm{C} 6$ levels (arrow). F: Right lateral three-dimensional $\mathrm{CT}$ angiogram revealing entry of the right thyrocervical trunk into the right $\mathrm{C} 4-5$ intervertebral foramen (arrowhead).
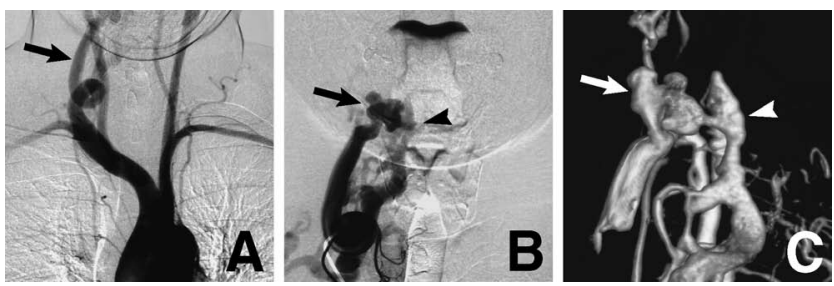

Fig. 2 Pretreatment digital subtraction angiography images. A: Aortic angiogram showing an enlarged right thyrocervical trunk (arrow), which is a single feeder. B, C: Right thyrocervical trunk angiograms showing a high flow paravertebral arteriovenous fistula (arrow) fed by the right thyrocervical trunk that drains into the epidural venous plexus (arrowhead) without reflux into the perimedullary veins.

was only to the epidural venous plexus, with no reflux into the perimedullary veins (Fig. 2B, C).

Transarterial embolization as the first therapeutic strategy was selected to reduce the compression to the spinal cord and nerve roots, and excessive bleeding during direct surgery. Under systemic heparinization, 5 French and 6 French guiding catheters were placed into the right thyrocervical trunk via the bilateral transfemoral approach, and 2 microcatheters (Excelsior SL-10 and Excelsior 1018; Boston Scientific, Natick, California, USA) were positioned in the fistula and the draining vein through the 2 guiding catheters for separate introduction of each
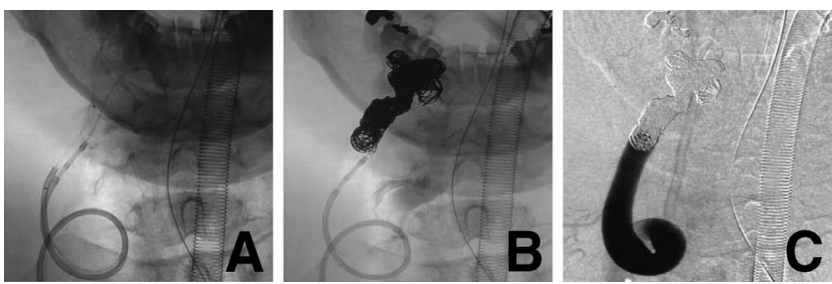

Fig. 3 A: Intraoperative digital subtraction angiography (DSA) image showing 2 microcatheters set in the fistula and the draining vein through 2 guiding catheters introduced into the right thyrocervical trunk. B, C: Posttreatment DSA images demonstrating complete occlusion of the fistula with no residual feeders after coil embolization.

microcatheter (Fig. 3A). We considered that embolization within a fistula did not adversely affect clinical outcome, and used coils because the radiculopathy was caused by compression of a dilated epidural venous plexus. A few large coils were initially positioned through the Excelsior 1018 microcatheter placed in the distal part of the fistulous draining venous side to prevent distal migration of additional coils. Further coils were then packed into the fistula containing the drainage side and subsequently packed into the dilated part of the feeder through the other microcatheter. Since the fistula was completely occluded and no residual feeders were present (Fig. 3B, C), the procedure was completed by only transarterial coil embolization. After the treatment, dilation of the superficial vein and bruit completely disappeared, and neurological symptoms gradually recovered. Six-month follow-up angiography after embolization showed no evidence of recanalization or new abnormal channels.

\section{Discussion}

Paravertebral AVF is a rare vascular malformation. The exact etiology is not well understood, but the reported association with neurofibromatosis type-1 (NF-1) and previous surgery or trauma ${ }^{13)}$ suggests an acquired lesion. However, the present patient had no history of health problems or trauma, and the cause was unclear. Fifty-eight percent of paravertebral AVF cases are found in the cervical region. ${ }^{7)}$ In addition, cervical lesions are the most common type complicated with NF-1, and typically originate from the vertebral artery. ${ }^{6}$ Paravertebral AVF can cause venous congestive myelopathy similar to that of spinal dural AVF,13) but is usually asymptomatic because a "reflux-impending mechanism," a substantial narrowing and bending of the vessel within the dural sleeves, interferes with retrograde flow of the arterialized epidural plexus into radicular or perimedullary veins. ${ }^{8)}$ Therefore, compressive radiculopathy or myelopathy as a result of an enlarged epidural venous plexus might be one of the first symptoms. ${ }^{5,10)}$

The therapeutic intention is to completely obliterate not only the fistula but also the draining vein. ${ }^{5)}$ The treatment strategy of paravertebral AVF, which mostly includes a high flow shunt ${ }^{3)}$ and multiple feeding arteries, ${ }^{13)}$ is en- 
dovascular embolization or direct surgery, but this strategy still remains controversial. We believe that endovascular surgery should be the first choice for the treatment of paravertebral AVF. Endovascular surgery is a less invasive treatment and may be useful as a preoperative adjunct in complex multifocal fistula. ${ }^{2)}$ By contrast, direct surgery interrupting both the shunting point and the origin of the draining vein is a reliable definitive therapy, but carries risks such as massive bleeding and obstruction of endovascular access that restricts further interventional radiology. ${ }^{6)}$ Direct surgery may be considered as a therapeutic option combined with embolization for cases with complex angioarchitecture. ${ }^{11)}$ Endovascular treatment is accomplished with liquid embolic agents, coils, detachable balloons, or a combination of these materials. ${ }^{10)}$ Transarterial embolization is commonly performed and is ideal for single fistula with a high flow. ${ }^{2,3}$ ) Transvenous embolization is beneficial for fistulas with multiple arterial feeders, and is less risky than the transarterial approach when considering the risk of anterior spinal artery occlusion. ${ }^{13}$ However, transvenous catheterization through the azygos vein and small epidural venous system is difficult. ${ }^{13)}$

Since our case was a high flow AVF fed by a single vessel and selective catheterization into the enlarged feeder was possible, transarterial embolization was planned. Moreover, we did not use the liquid agents such as n-butylcyanoacrylate to prevent the risks of migration into the intracranial circulation via aberrant anastomoses ${ }^{4)}$ and unintentional occlusion of the drainage veins. Several technical adjuncts including proximal temporary balloon occlusion for flow control are important for successful embolization. ${ }^{10)}$ Covered stent placement can preserve vessels during the treatment of large AVFs fed by the vertebral artery. ${ }^{12)} \mathrm{A}$ procedure using 2 microcatheters such as in our case is also useful. A large and high flow fistula requires that the stability of the detached coil must be maintained. Coils in the drainage side deposited through the arterial route prevent distal coil migration, and secure accurate embolization is facilitated. In the present case, complete obliteration of the AVF was obtained without complications. Endovascular surgery that can be treated using several adjunctive procedures may be a safe and effective treatment modality for paravertebral AVF.

\section{References}

1) Bao YH, Ling F: Classification and therapeutic modalities of spinal vascular malformations in 80 patients. Neurosurgery
40: 75-81, 1997

2) Chuang NA, Shroff MM, Willinsky RA, Drake JM, Dirks PB, Armstrong DC: Slow-flow spinal epidural AVF with venous ectasias: two pediatric case reports. AJNR Am J Neuroradiol 24: 1901-1905, 2003

3) Chul Suh D, Gon Choi C, Bo Sung K, Kim KK, Chul Rhim S: Spinal osseous epidural arteriovenous fistula with multiple small arterial feeders converging to a round fistular nidus as a target of venous approach. AJNR Am J Neuroradiol 25: 69-73, 2004

4) Geibprasert S, Pongpech S, Armstrong D, Krings T: Dangerous extracranial-intracranial anastomoses and supply to the cranial nerves: vessels the neurointerventionalist needs to know. AJNR Am J Neuroradiol 30: 1459-1468, 2009

5) Goyal M, Willinsky R, Montanera W, terBrugge K: Paravertebral arteriovenous malformations with epidural drainage: clinical spectrum, imaging features, and results of treatment. AJNR Am J Neuroradiol 20: 749-755, 1999

6) Hauck EF, Nauta HJ: Spontaneous spinal epidural arteriovenous fistulae in neurofibromatosis type-1. Surg Neurol 66: 215-221, 2006

7) Kawabori M, Hida K, Yano S, Asano T, Iwasaki Y: Cervical epidural arteriovenous fistula with radiculopathy mimicking cervical spondylosis. Neurol Med Chir (Tokyo) 49: 108-113, 2009

8) Krings T, Mull M, Bostroem A, Otto J, Hans FJ, Thron A: Spinal epidural arteriovenous fistula with perimedullary drainage. Case report and pathomechanical considerations. J Neurosurg Spine 5: 353-358, 2006

9) Krings T, Mull M, Gilsbach JM, Thron A: Spinal vascular malformations. Eur Radiol 15: 267-278, 2005

10) Niimi Y, Berenstein A, Fernandez PM, Brisman JL, Song JK: Pediatric nonvertebral paraspinal arteriovenous fistulas along the segmental nerve: clinical, imaging, and therapeutic considerations. J Neurosurg 103: 156-162, 2005

11) Pirouzmand F, Wallace MC, Willinsky R: Spinal epidural arteriovenous fistula with intramedullary reflux. Case report. $J$ Neurosurg 87: 633-635, 1997

12) Sadato A, Satow $T$, Ishii $A$, Takayama $M$, Hashimoto $N$ : Large vertebral arteriovenous fistula treated with stentgrafts-case report. Neurol Med Chir (Tokyo) 43: 250-254, 2003

13) Silva N Jr, Januel AC, Tall P, Cognard C: Spinal epidural arteriovenous fistulas associated with progressive myelopathy. Report of four cases. J Neurosurg Spine 6: 552-558, 2007

Address reprint requests to: Associate Professor Hidenori Oishi, MD, PhD, Department of Neurosurgery, Juntendo University School of Medicine, 2-1-1 Hongo, Bunkyo-ku, Tokyo 113-8431, Japan. e-mail: oishi@juntendo.ac.jp 